\title{
Efficacy and Safety of Alogliptin in Patients With Type 2 Diabetes: Analysis of the ATTAK-J Study
}

\author{
Hiroshi Takeda ${ }^{\mathrm{a}}$, Nobuo Sasai ${ }^{\mathrm{a}}$, Shogo Ito ${ }^{\mathrm{a}}$, Mitsuo Obana ${ }^{\mathrm{a}}$, Tetsuo Takuma ${ }^{\mathrm{a}}$, Masahiko Takai ${ }^{\mathrm{a}}$, \\ Hideaki Kaneshige $^{\mathrm{a}}$, Hideo Machimura ${ }^{\mathrm{a}}$, Akira Kanamori ${ }^{\mathrm{a}}$, Kazumi Nakajima ${ }^{\mathrm{b}}$, Ikuro Matsuba ${ }^{\mathrm{a}}$,
}

\begin{abstract}
Background: Dipeptidyl peptidase-4 (DPP-4) inhibitors have been shown to reduce hemoglobin A1c (HbAlc) in patients with type 2 diabetes, but the reduction varies between patients and adequate glycemic control may not be achieved. We evaluated the efficacy and safety of the DPP-4 inhibitor alogliptin in the real clinical setting, and analyzed factors associated with the improvement of $\mathrm{HbA} 1 \mathrm{c}$ by alogliptin treatment.
\end{abstract}

Methods: A retrospective observational study was performed in patients with type 2 diabetes attending hospitals or clinics belonging to the Kanagawa Physicians Association who received treatment with alogliptin for 1 year or longer. Patients using insulin were excluded from the study. The efficacy endpoints were HbA1c (National Glycohemoglobin Standardization Program value), blood glucose (fasting/ postprandial), body weight, blood pressure (systolic/diastolic), liver function (glutamate oxaloacetate transaminase, glutamate pyruvate transaminase, and $\gamma$-glutamyl transpeptidase), kidney function (serum creatinine and estimated glomerular filtration rate), serum lipids (total cholesterol, low-density lipoprotein cholesterol, high-density lipoprotein cholesterol, and triglycerides), and serum amylase. Adverse events were compiled to assess safety.

Results: Of 330 patients whose case records were collected, 27 patients were excluded for protocol violations, leaving 303 patients to form the full analysis set. Compared with baseline, HbAlc showed a decrease by $0.54 \pm 1.22 \%$ (mean \pm standard deviation) after 12 months of alogliptin treatment. Factor analysis demonstrated that the change of $\mathrm{HbAlc}$ after 12 months was significantly influenced by the baseline HbA1c level, duration of diabetes, concomitant use of sulfonylureas, and compliance with diet therapy. In addition, there was a significant reduction of total cholesterol, low-density lipoprotein cholesterol, and the estimated glomerular filtration rate after 12 months of alogliptin

Manuscript accepted for publication December 04, 2015

aDiabetes Committee Study Group, Kanagawa Physicians Association, Kanagawa, Japan

bNakajima Clinic, Kanagawa, Japan

${ }^{\mathrm{c} C o r r e s p o n d i n g ~ A u t h o r: ~ I k u r o ~ M a t s u b a, ~ M a t s u b a ~ C l i n i c, ~ 2-159 ~ T s u k a g o s h i, ~}$ Saiwai-ku, Kawasaki-shi, Kanagawa 212-0024, Japan.

Email: ikuro@matsuba-web.com

doi: http://dx.doi.org/10.14740/jocmr2418w treatment, as well as a significant increase in serum creatinine. No significant changes of body weight, blood pressure, or liver function were observed. Symptoms of hypoglycemia occurred in two patients $(0.6 \%)$.

Conclusions: Alogliptin displayed a significant hypoglycemic effect and excellent safety in routine clinical use. Factors influencing the change of HbA1c with alogliptin therapy may include the HbA1c at the start of treatment, the duration of diabetes, use of sulfonylureas, and compliance with diet therapy.

Keywords: Type 2 diabetes; Dipeptidyl peptidase-4 inhibitor; Alogliptin; Hemoglobin A1c

\section{Introduction}

Dipeptidyl peptidase-4 (DPP-4) inhibitors are a new class of oral antidiabetic agents that increase endogenous incretin levels and stimulate glucose-dependent insulin secretion by selectively inhibiting DPP-4, an enzyme that degrades circulating incretins (glucagon-like peptide-1 and glucose-dependent insulinotropic polypeptide) [1]. In 2009, sitagliptin was the first of these drugs to be approved in Japan and eight DPP-4 inhibitors are available as of 2015. Meta-analyses have shown that there is no significant difference of the hypoglycemic effect between DPP-4 inhibitors $[2,3]$. These drugs have a good safety profile with a low risk of causing hypoglycemia or weight gain [4].

Alogliptin is a DPP-4 inhibitor that was marketed in Japan in 2010 [5]. A meta-analysis of the efficacy of alogliptin showed that hemoglobin A1c (HbA1c) was decreased by $0.81 \%$ (at a dose of $12.5 \mathrm{mg}$ ) and by $0.98 \%$ (at a dose of 25.0 $\mathrm{mg}$ ) in patients treated with this drug compared with controls [6]. In addition, a large-scale comparative study (the EXAMINE study) found no difference in the risk of cardiovascular events between alogliptin and placebo group in patients with type 2 diabetes mellitus (T2DM) who had a history of acute coronary syndrome [7].

While DPP-4 inhibitors reduce $\mathrm{HbA} 1 \mathrm{c}$, the extent of the reduction varies between patients and some patients do not achieve adequate glycemic control. A meta-analysis of factors associated with $\mathrm{HbAlc}$ reduction indicated that baseline $\mathrm{HbAlc}$ and fasting blood glucose levels were useful predictors of the response [8]. Additionally, a meta-analysis of racial 


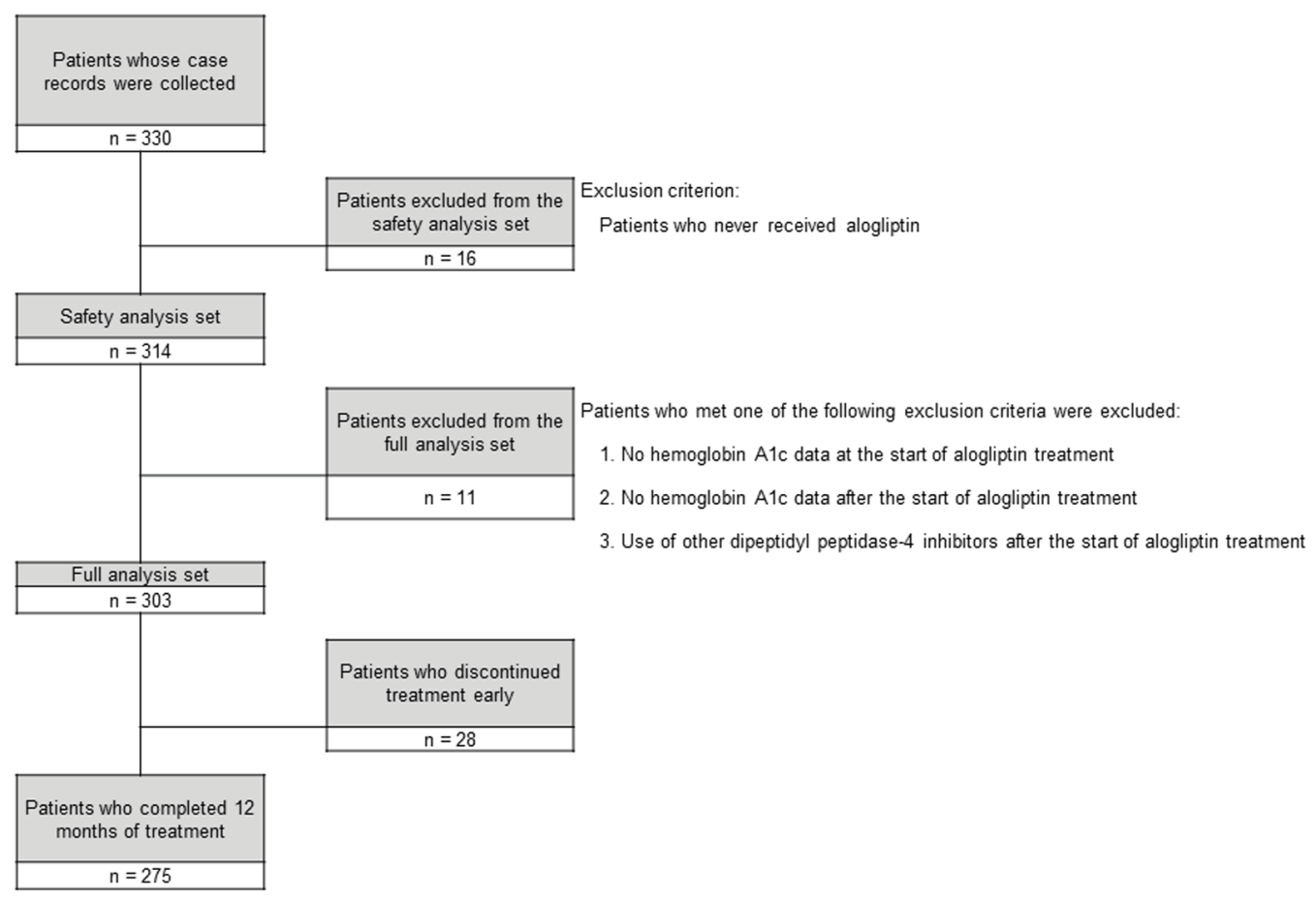

Figure 1. Disposition of the subjects.

differences revealed that the reduction of HbA1c by DPP-4 inhibitors was greater in Asians than in non-Asians, and that body mass index (BMI) had a significant influence [9].

It has been reported that DPP-4 inhibitors can have lipidlowering [10] and renoprotective [11] effects, in addition to their hypoglycemic effect. However, there were no significant difference in the changes of the lipid profile or estimated glomerular filtration rate (eGFR) between alogliptin and placebo in the EXAMINE study [7].

In order to evaluate the efficacy and safety of alogliptin in the real clinical setting, a retrospective observational study was conducted for 1 year from the start of alogliptin treatment in patients with T2DM who were attending clinics/hospitals belonging to the Kanagawa Physicians Association. The primary efficacy endpoint was the change of HbAlc after 12 months of treatment, and factor analysis was performed to identify patient characteristics associated with the improvement of HbA1c that could be used to predict efficacy.

\section{Patients and Methods}

\section{Study design}

A multicenter retrospective observational study was conducted at clinics and hospitals belonging to the Kanagawa Physicians Association. Data were collected from the medical records of the subjects and the follow-up period was 1 year. This study was approved by the Ethics Review Board of the Kanagawa Physicians Association.

\section{Patients}

Patients were eligible for this study if they had T2DM, were aged 20 years old or older, regularly attended a clinic or hospital belonging to the Kanagawa Physicians Association, and received treatment with alogliptin for 1 year or longer. Alogliptin was started if glycemic control was inadequate for at least 1 month despite diet and exercise therapy or diet and exercise plus oral antidiabetic drugs.

The exclusion criteria were as follows: a history of hypersensitivity to any component of alogliptin; a history of severe ketoacidosis, diabetic coma or precoma within 6 months before the start of alogliptin therapy; severe infection; recent or planned surgery or severe trauma; concurrent use of insulin preparations or glinides; and patients who the attending doctor considered to be inappropriate for this study for other reasons.

\section{Items investigated}

The baseline characteristics investigated for the subjects included the gender, age, height, duration of diabetes, family history, smoking history, alcohol history, and complications. Use of the following drugs was assessed before treatment with alogliptin, at the start of treatment, and 3 months, 6 months, 9 months, and 12 months after the start of treatment: alogliptin and other antidiabetic drugs, lipid-lowering drugs, and antihypertensive drugs. Efficacy endpoints were determined at each of the specified times, including HbA1c (National Gly- 
cohemoglobin Standardization Program value), blood glucose (fasting and postprandial), body weight (BW), blood pressure (BP; systolic/diastolic), liver function parameters (glutamate oxaloacetate transaminase, glutamate pyruvate transaminase, and $\gamma$-glutamyl transpeptidase), kidney function parameters (serum creatinine, eGFR), serum lipids (total cholesterol (TC), low-density lipoprotein (LDL) cholesterol, high-density lipoprotein cholesterol, triglycerides (TG)), and serum amylase. In addition, adverse events were evaluated at each of these times to assess safety.

\section{Statistical analysis}

After patients who did not receive the study drug were excluded from those whose case records were collected, the remaining patients formed the safety analysis set. The full analysis set (FAS) was obtained by excluding patients who met any of the following criteria from the safety analysis set: 1) no HbAlc data at the start of alogliptin treatment, 2) no HbA1c data after the start of alogliptin treatment, or 3) administration of another DPP-4 inhibitor after the start of alogliptin treatment.

Appropriate descriptive statistics were calculated for the baseline characteristics of the FAS, including the gender, age, duration of diabetes, BMI, and BP.

Descriptive statistics were also calculated for the daily dose of alogliptin at each time of assessment, and for the use or non-use of other antidiabetic drugs, lipid-lowering drugs, and antihypertensive drugs at each assessment time.

Furthermore, descriptive statistics were calculated for all of the efficacy endpoints at each time of assessment. The following statistical analyses were performed. Linear mixed-effects models for repeated measures were employed to evaluate differences between each assessment time compared with the start of alogliptin treatment using the time of assessment as the fixed effect. Adjustment for multiplicity due to the number of assessment time points was done by the Dunnett-Hsu method. The percentage of patients who achieved specific HbAlc target values $(<6.0 \%,<7.0 \%$, or $<8.0 \%)$ was calculated at each time of assessment, and McNemar's test was used to evaluate differences in the percentage at each time compared with the start of alogliptin treatment. Two multiple regression analysis models, which were a model using five baseline characteristics as explanatory variables (model 1) and a model using those variables plus concurrent treatment (in month 12) as the variables (model 2), were employed to analyze factors associated with the change of HbA1c (in month 12).

In the safety analysis set, the number and percentage of patients who developed adverse events (symptoms of hypoglycemia, constipation, and other events) and the number of episodes of each adverse event were tallied.

\section{Results}

\section{Disposition of the subjects}

The disposition of the subjects is shown in Figure 1. Of 330 patients whose case records were collected, 16 patients who did not receive alogliptin were excluded and the remaining 314 patients formed the safety analysis set. Then 11 patients who met any of the exclusion criteria for the FAS were excluded from those in the safety analysis set, leaving 303 patients in the FAS. Of these 303 patients, 28 patients discontinued alogliptin early and 275 patients completed 12 months of treatment.

\section{Baseline characteristics}

Table 1 provides a summary of the baseline characteristics of the patients in the FAS $(n=303)$. Their mean age was 67.3 years and there was male predominance $(56.1 \%)$. The mean duration of diabetes was 10.3 years and the diabetic complications included retinopathy (7.6\%), neuropathy $(8.6 \%)$, and nephropathy $(11.2 \%)$. Regarding other complications, hypertension (59.4\%) and dyslipidemia (55.8\%) showed a high prevalence.

\section{Medications}

The medications used by the patients in the FAS $(\mathrm{n}=303)$ are shown in Table 2. Before the start of treatment with alogliptin, $74.3 \%$ of the patients were using one or more antidiabetic drugs. Drugs taken by $10 \%$ or more of the patients included glimepiride (31.0\%), other DPP-4 inhibitors (26.4\%), $\alpha$-glucosidase inhibitors (24.8\%), metformin (24.1\%), and pioglitazone (19.5\%). At the start of alogliptin treatment, the patients were not using other DPP-4 inhibitors and the percentage of patients using $\alpha$-glucosidase inhibitors or glinides showed a marked decrease from $24.8 \%$ to $15.8 \%$ and from $5.9 \%$ to $0.3 \%$, respectively. On the other hand, the use of metformin increased after the start of alogliptin treatment and $34.9 \%$ of the patients were taking it after 12 months.

Before the start of alogliptin treatment, $43.9 \%$ of the patients were taking lipid-lowering drugs and 55.4\% were taking antihypertensive agents, with these percentages increasing to $52.0 \%$ and $63.3 \%$, respectively, at 12 months after the start of alogliptin treatment.

The mean daily dose of alogliptin is displayed in Figure 2 (left). It was $23.7 \mathrm{mg}$ at the start of treatment and did not change significantly, being $23.8 \mathrm{mg}$ after 12 months of treatment.

\section{Changes of HbA1c}

The changes of HbAlc are shown in Figure 3. Mean HbAlc decreased from $7.37 \%$ at the start of alogliptin treatment to $6.78 \%$ after 6 months of treatment and did not change much after that, being $6.83 \%$ at 12 months. After 12 months of alogliptin treatment, $\mathrm{HbA} 1 \mathrm{c}$ (mean \pm standard deviation) was reduced by $0.54 \pm 1.22 \%(\mathrm{n}=259)$.

The percentage of patients achieving the target HbA1c level was significantly larger at every time of assessment from 3 months after the start of alogliptin treatment compared with before alogliptin treatment (HbA1c was $<7.0 \%$ in $42.2 \%$ of 
Table 1. Baseline Characteristics of the Full Analysis Set $(N=303)$

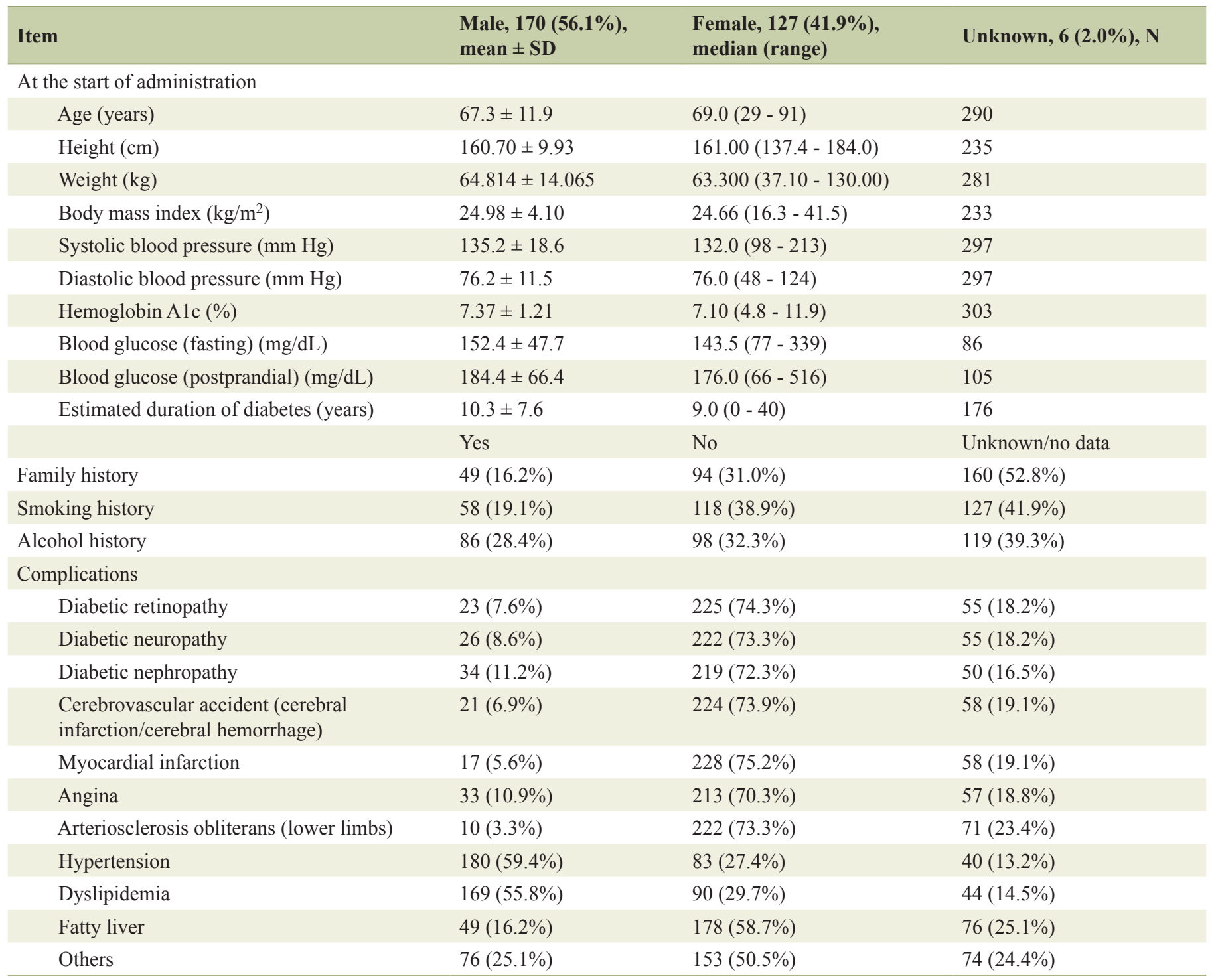

patients before alogliptin treatment vs. $63.3 \%$ after 12 months; $\mathrm{P}<0.001$ by McNemar's test).

The results of multiple regression analysis using the change of $\mathrm{HbAlc}$ after 12 months as a response variable are displayed in Table 3. Model 1 (using baseline characteristics as the explanatory variables) was employed for analysis of 110 patients from the FAS with complete data on the age, disease duration, and BMI. This analysis showed that the disease duration and the baseline HbAlc level had a significant influence on the change of HbA1c after 12 months. The reduction of $\mathrm{HbA} 1 \mathrm{c}$ was larger as the disease duration became shorter and as baseline $\mathrm{HbA} 1 \mathrm{c}$ increased.

Then analysis was performed using model 2 (adding concurrent treatment to the explanatory variables of model 1 ) in the patients from model 1 with information on concurrent treatment at 12 months. This analysis showed that use or non-use of sulfonylureas at 12 months and compliance with diet therapy significantly influenced the change of $\mathrm{HbAlc}$, in addition to the influence of baseline HbA1c. When sulfonylureas were not being used after 12 months and compliance with diet was better, the reduction of $\mathrm{HbA} 1 \mathrm{c}$ at 12 months was larger. While the duration of diabetes had a significant influence on $\mathrm{HbA1c}$ in model 1, there was no significant effect in model 2.

\section{Other efficacy endpoints}

Changes of BW over time in the FAS are shown in Figure 2 (right). Mean BW did not change significantly, being $64.95 \mathrm{~kg}$ before the start of alogliptin treatment and $65.10 \mathrm{~kg}$ after 12 months of treatment.

The results for the other efficacy endpoints are listed in Tables 4-6. After 12 months of alogliptin treatment, serum 
Table 2. Medications of the Full Analysis Set

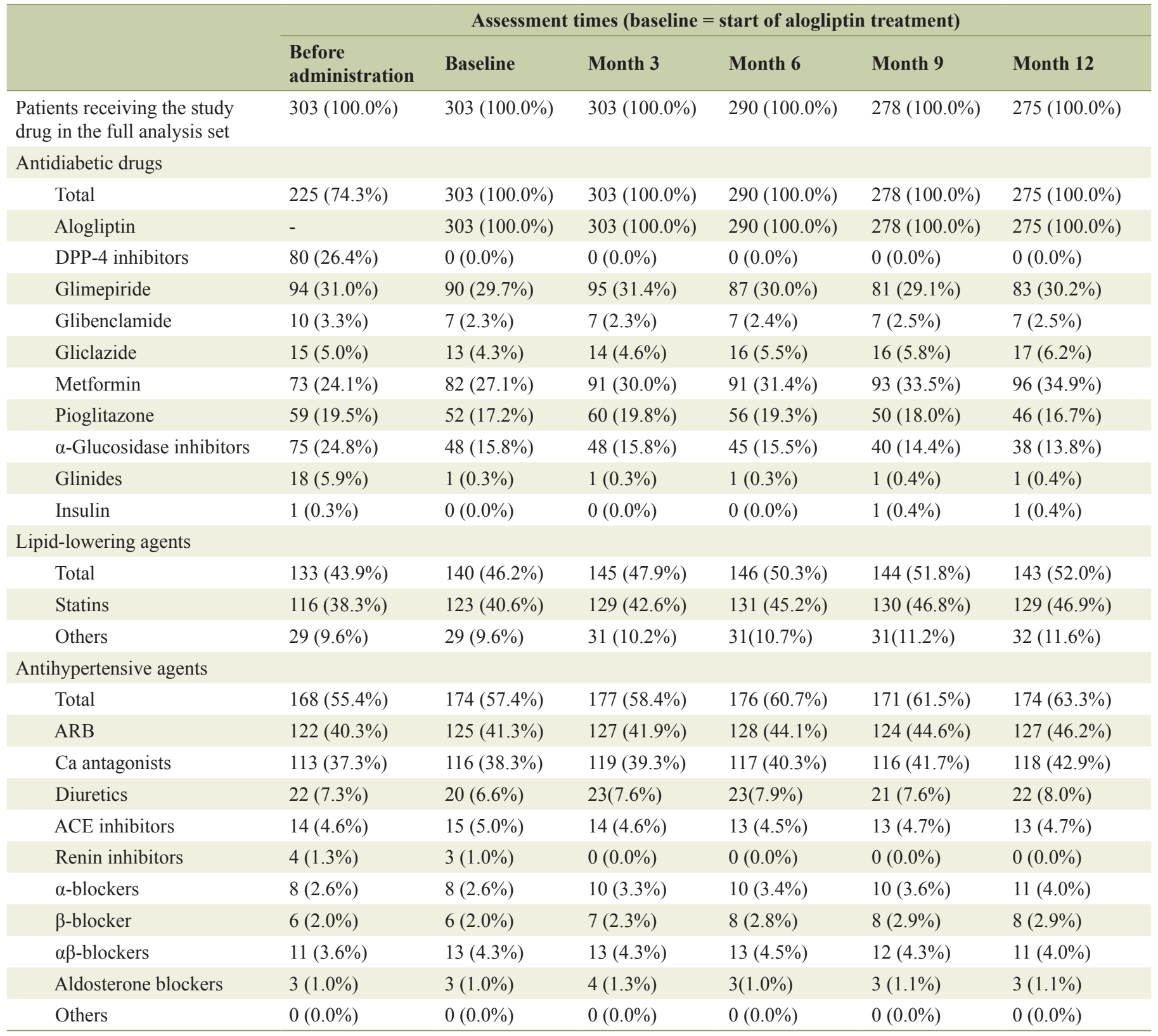

ACE: angiotensin-converting enzyme; ARB: angiotensin receptor blocker; DPP-4: dipeptidyl peptidase-4.

creatinine showed a significant increase, while fasting blood glucose, eGFR, TC, and LDL cholesterol were all significantly decreased.

\section{Safety}

Twelve adverse events were reported in eight out of 314 patients $(2.5 \%)$ in the safety analysis set. These adverse events included constipation (six events in three patients), hypoglycemia (two events in two patients), and fracture, neuropathy, hypertension, and lipid abnormality (each event occurred in one patient).

\section{Discussion}

The present study investigated the efficacy and safety of alogliptin therapy in patients with T2DM who were attending hospitals or clinics belonging to the Kanagawa Physicians Association, employing efficacy endpoints such as the profile of $\mathrm{HbAlc}$ over time or the numerical change of HbAlc.

Of the 330 patients whose case records were collected, 27 patients were excluded from the FAS. Thus, a high propor- 

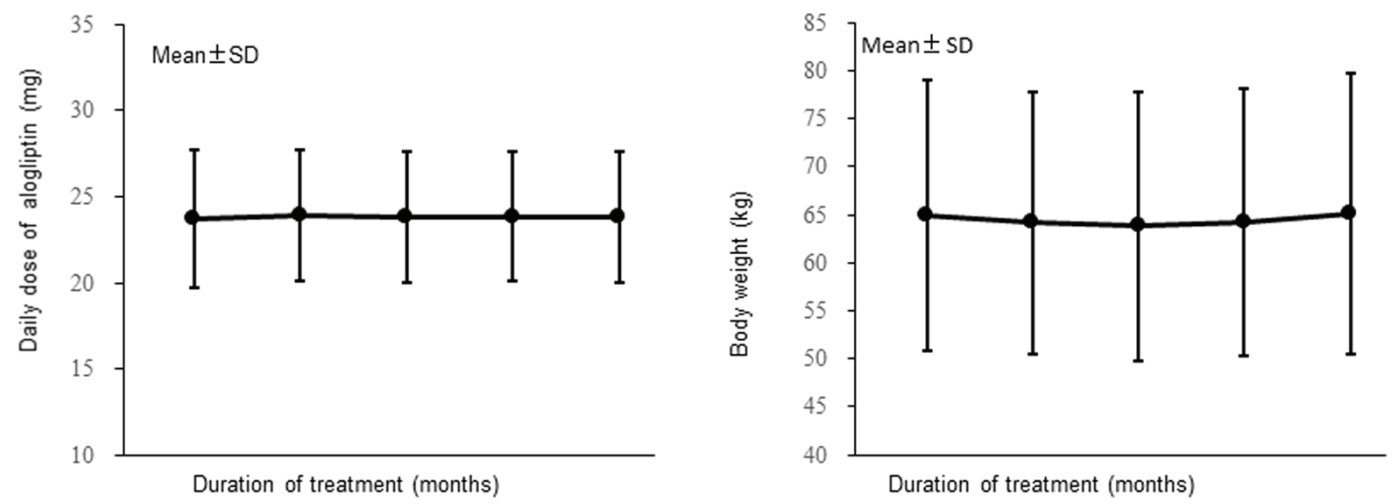

Figure 2. Changes of the daily dose of alogliptin and the body weight (full analysis set).

tion of all subjects were included in the FAS $(91.8 \% ; 303 / 330$ patients).

Comparison of baseline characteristics between this study and a study of sitagliptin conducted in 1,332 patients [11] revealed that the sex ratio and BMI were similar $(56.1 \%$ men in this study vs. $56.4 \%$ and mean BMI of 24.98 vs. 24.6), but the mean age (67.3 vs. 62.9 years old), mean systolic BP (135.2 $\mathrm{mm} \mathrm{Hg}$ vs. $128.5 \mathrm{~mm} \mathrm{Hg}$ ), proportion of patients with hypertension $(59.4 \%$ vs. $32 \%)$, and proportion of patients with dyslipidemia (55.8\% vs. $36 \%$ ) were higher in the present study. On the other hand, the mean duration of diabetes (10.3 vs. 12.0 years), mean $\mathrm{HbA} 1 \mathrm{c}(7.37 \%$ vs. $8.0 \%)$, and proportion of patients with complications of diabetes (retinopathy, $7.6 \%$ vs. $32 \%$; neuropathy, $8.6 \%$ vs. $26 \%$; nephropathy, $11.2 \%$ vs. $28 \%$ ) were lower in this study. Thus, compared with the patient population of the sitagliptin study, the age, BP, and lipid levels were higher and glycemic control was better at the start of alogliptin treatment in this study, while fewer patients had diabetic complications.

Before the start of alogliptin treatment, $74.3 \%$ of the patients were using antidiabetic drugs, including glimepiride (31.0\%), other DPP-4 inhibitors $(26.4 \%), \alpha$-glucosidase inhibitors ( $\alpha$-GIs) $(24.8 \%)$, metformin $(24.1 \%)$, and pioglitazone $(19.5 \%)$. At the start of alogliptin treatment, $39.3 \%$ of the patients were not taking concomitant drugs, $34.0 \%$ were

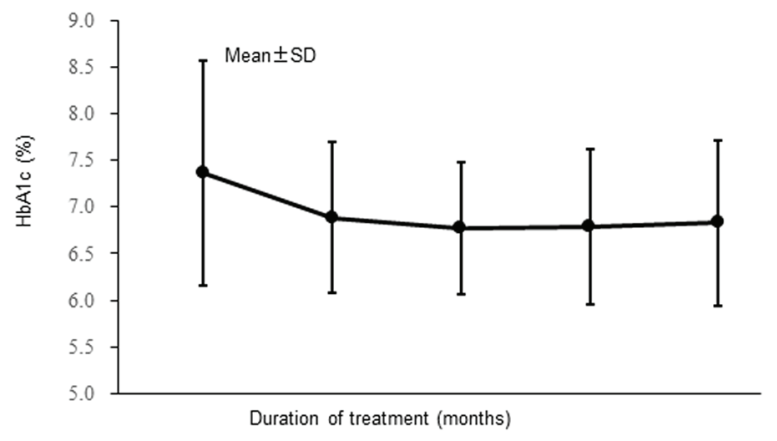

Figure 3. Changes of hemoglobin A1c (full analysis set). taking one drug, $17.5 \%$ were using two drugs, and $9.2 \%$ were on three drugs. The concomitant drugs included glimepiride $(29.7 \%)$, metformin $(27.1 \%)$, pioglitazone $(17.2 \%)$, and $\alpha$-GIs (15.8\%). After 12 months of alogliptin treatment, $34.0 \%$ of the patients were not taking concomitant drugs, $34.5 \%$ were taking one drug, $24.0 \%$ were using two drugs, $6.5 \%$ were using three drugs, and $0.7 \%$ were on four drugs. Concomitant drugs included metformin $(34.9 \%)$, glimepiride $(30.2 \%)$, pioglitazone $(16.72 \%)$, and $\alpha$-GIs $(13.8 \%)$.

HbA1c decreased significantly over time after the start of alogliptin treatment, and the mean reduction of $\mathrm{HbA} 1 \mathrm{c}$ at 12 months was $0.54 \%$. In a study of 1,057 patients, the mean reduction of $\mathrm{HbA} 1 \mathrm{c}$ after 12 months of treatment with sitagliptin was reported to be $0.7 \%[12,13]$. The reduction of $\mathrm{HbA} 1 \mathrm{c}$ was smaller in the present study, probably because baseline HbA1c levels were different (mean baseline HbA1c was $7.37 \%$ in this study vs. $8.0 \%$ in the sitagliptin study).

Multivariate analysis was performed using baseline characteristics as the explanatory variables (model 1) to identify factors associated with the change of $\mathrm{HbAlc}$ after 12 months, revealing that the baseline $\mathrm{HbA} 1 \mathrm{c}$ and duration of diabetes had a significant independent influence on the change of HbAlc. Analysis was also performed after adding concurrent treatment to the explanatory variables (model 2), and identified concomitant use of sulfonamides and compliance with diet therapy as significant factors. In a previous study of 93 patients treated with sitagliptin, factor analysis revealed that noncompliance with diet therapy or exercise therapy were independent factors influencing the increase in HbA1c (by $0.3 \%$ or more) after 1.5 years [14].

In the present study, there were no significant changes of BW, BMI, BP (systolic/diastolic), and liver function (glutamate oxaloacetate transaminase, glutamate pyruvate transaminase, and $\gamma$-glutamyl transpeptidase) during alogliptin treatment. On the other hand, significant changes of lipids (TC and LDL cholesterol), kidney function (serum creatinine and eGFR), and the fasting blood glucose level were noted after 12 months of alogliptin treatment. In a study of 940 patients, TC showed a significant decrease and serum creatinine was increased significantly by sitagliptin treatment [15]. In addition, 


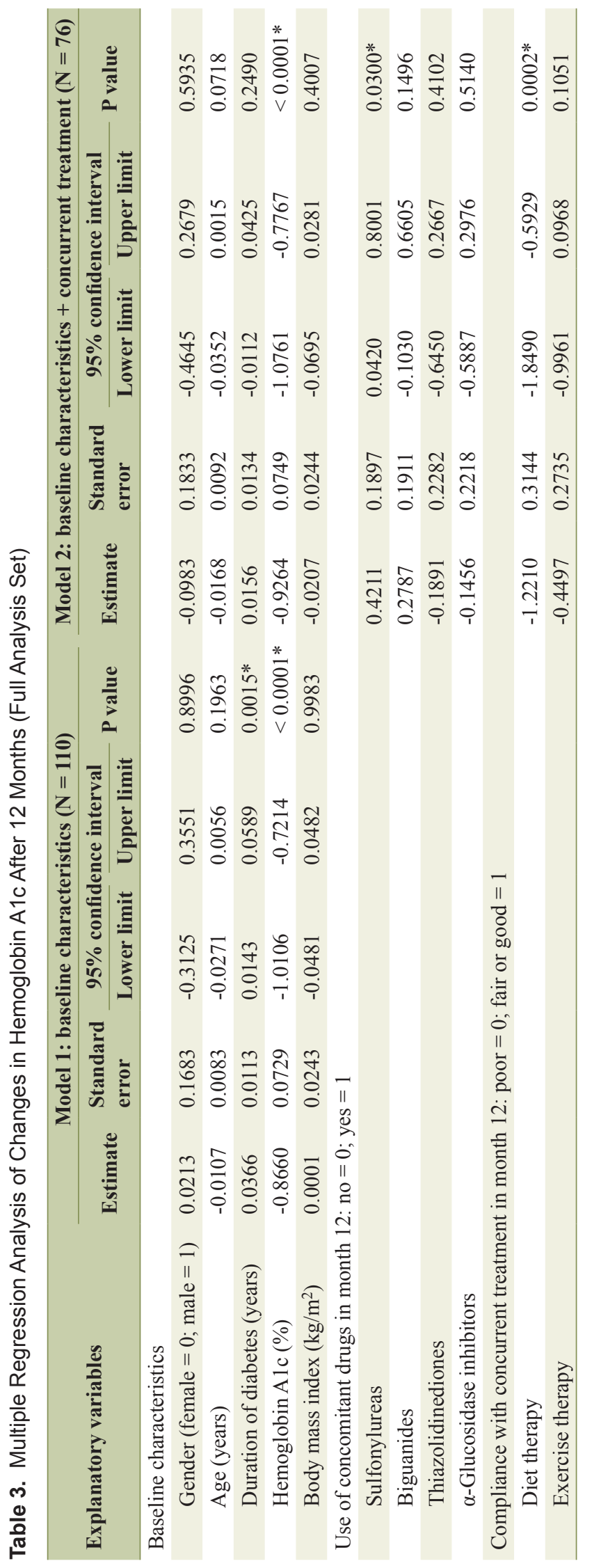

evaluation of serum creatinine up to 2 years $(n=826)$ showed that the serum creatinine level was increased significantly at 1 month after the start of sitagliptin treatment, but there were no significant changes subsequently [16].

There were 314 patients in the safety analysis set of the present study. The incidence rates of hypoglycemic symptoms and constipation were $0.6 \%(\mathrm{n}=2)$ and $1.0 \%(\mathrm{n}=3)$, respectively, so the data demonstrated that alogliptin was a very safe drug.

With regard to limitations, this was an observational study without a control group. When interpreting the results of the study, it should be noted that changes observed after the start of alogliptin treatment were not necessarily due to the effects of alogliptin.

\section{Conclusion}

$\mathrm{HbA1c}$ and fasting blood glucose levels were significantly reduced by treatment with alogliptin, demonstrating that this drug had a clinically useful hypoglycemic effect. Analysis of the changes of HbAlc suggested that the baseline HbAlc level, duration of diabetes, concomitant use of sulfonylureas, and compliance with diet therapy influenced the reduction of HbAlc by alogliptin. In addition, there was a significant reduction of TC and LDL cholesterol levels, suggesting that alogliptin had a lipid-lowering effect. Furthermore, a significant increase in serum creatinine and significant reduction of eGFR suggested that alogliptin influences kidney function. Alogliptin treatment was safe, with little change of BW and a low incidence of hypoglycemia $(0.6 \%)$.

\section{Acknowledgement}

The ATTAK-J study was performed by the diabetes committee study group of the Kanagawa Physicians Association. We thank the doctors and staff of the Kanagawa Physicians Association for cooperating with this research, and the patients for their participation in the study. The following hospital/clinics and investigators from the Kanagawa Physicians Association participated in this study: Sakura T's Clinic (Dr. Toshiaki Sakurazawa); Sakurai Clinic (Dr. Atsuko Sakurai); Mineki Naika Clinic (Dr. Hitoshi Mineki); Nippon Yakin Kogyo Clinic (Dr. Mitsuyuki Taira); Hayashi Diabetic Medicine Clinic (Dr. Tsutomu Hayashi); Machida Gastroenterology and Surgery Clinic (Dr. Tomoyuki Machida); Hara Clinic (Dr. Yoshikuni Hara); Shonan Chuo Clinic (Dr. Atsunori Oishi); Aikawa Hokubu Hospital (Dr. Hironori Adachi); Kubota Clinic (Dr. Takahiro Kubota); Hashimoto Clinic (Dr. Kenji Sakurai); Minami-guchi Clinic, Kuzuhadai Hospital (Dr. Takayuki Furuki); Suzuki Clinic (Dr. Shin Suzuki); Kikuchi Surgery and Internal Medicine Clinic (Dr. Hiroshi Kikuchi).

\section{Grant Support}

This study was funded by the Japan Diabetes Foundation. 


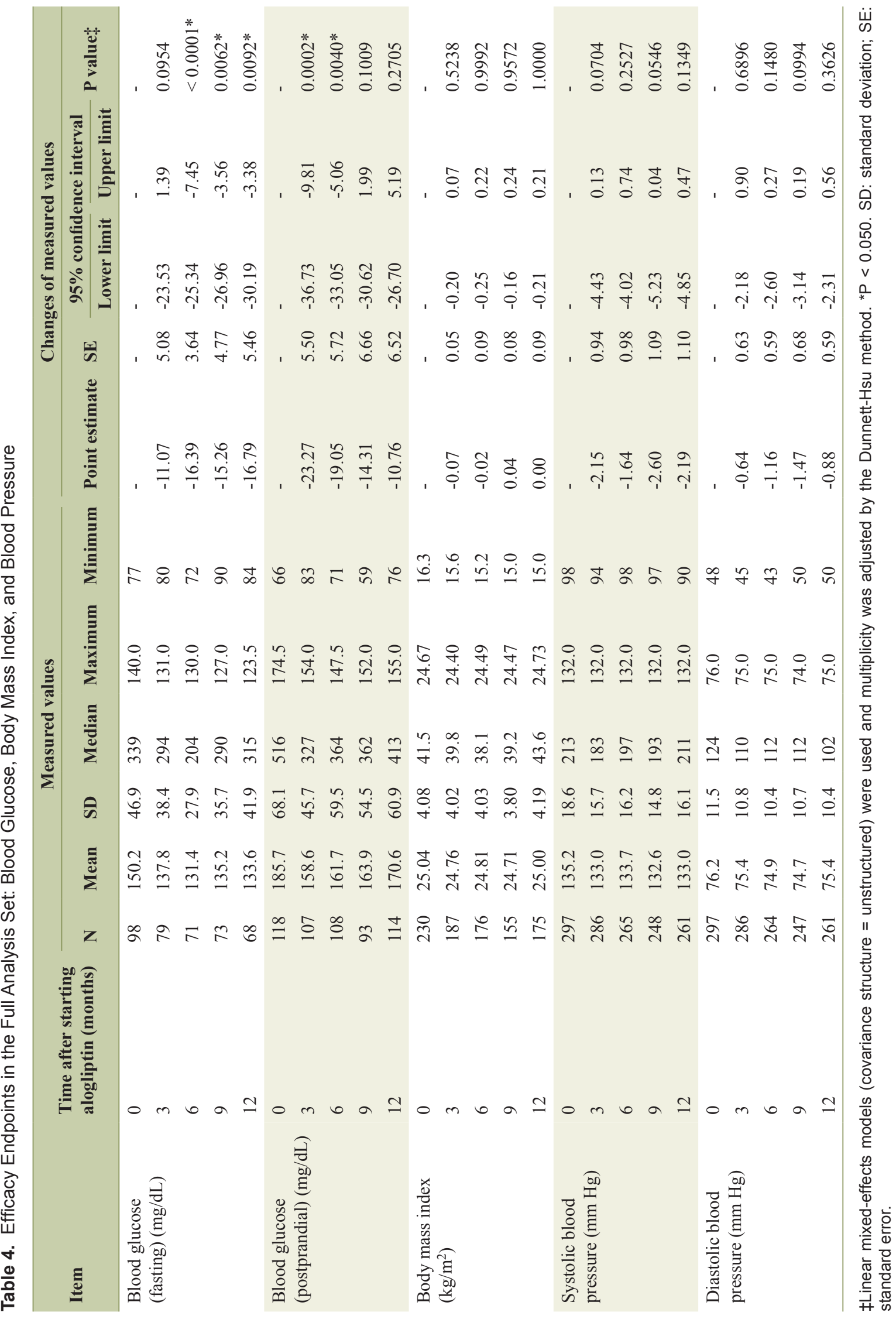




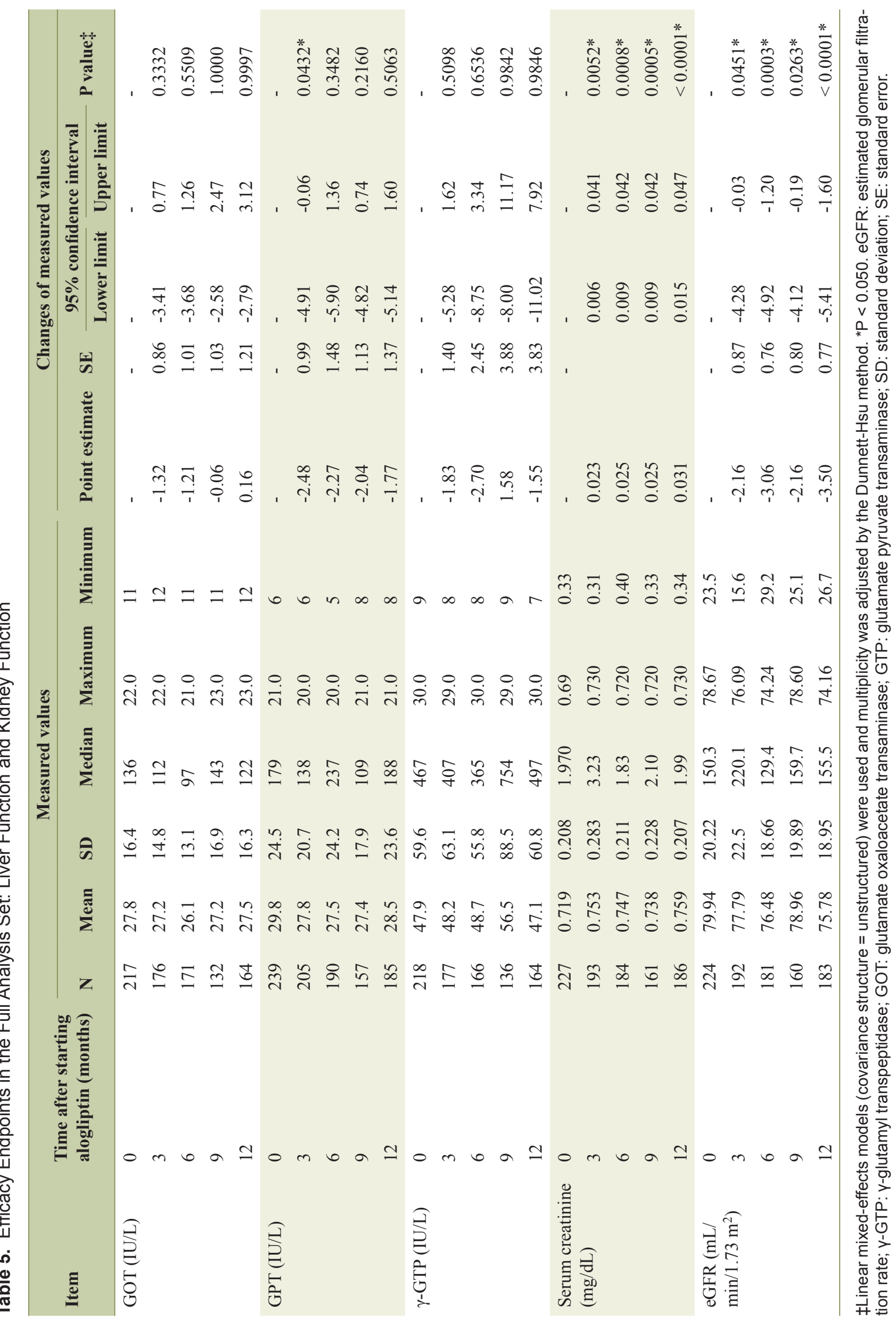




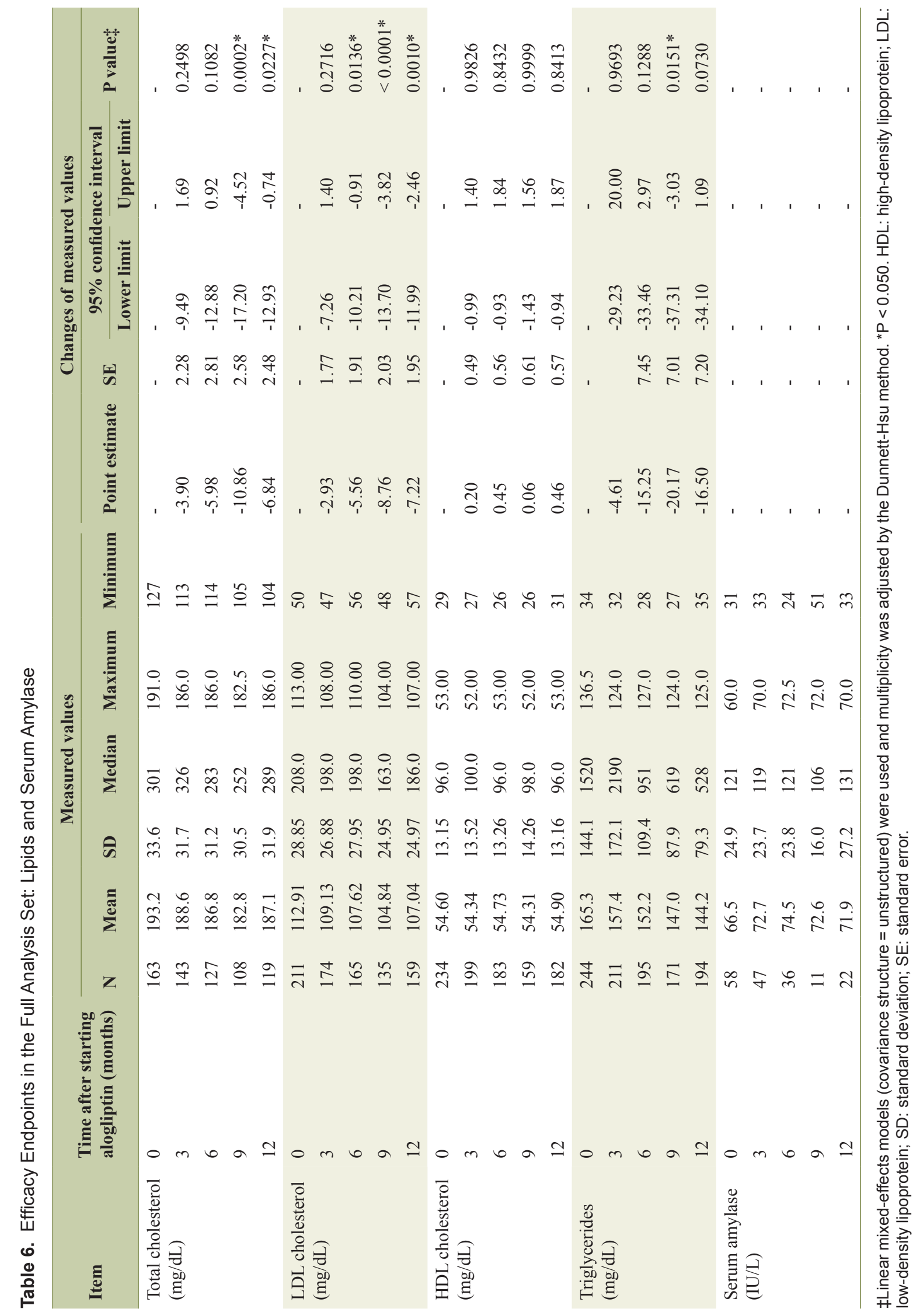




\section{Conflict of Interest}

This study was designed and planned by members of the Kanagawa Physicians Association and was funded by the Japan Diabetes Foundation. No company was involved in designing the study, patient enrollment, calculation or analysis of the data, interpretation of the findings, or writing the manuscript.

\section{References}

1. Scheen AJ. A review of gliptins for 2014. Expert Opin Pharmacother. 2015;16(1):43-62.

2. Aroda VR, Henry RR, Han J, Huang W, DeYoung MB, Darsow T, Hoogwerf BJ. Efficacy of GLP-1 receptor agonists and DPP-4 inhibitors: meta-analysis and systematic review. Clin Ther. 2012;34(6):1247-1258 e1222.

3. Craddy P, Palin HJ, Johnson KI. Comparative effectiveness of dipeptidylpeptidase-4 inhibitors in type 2 diabetes: a systematic review and mixed treatment comparison. Diabetes Ther. 2014;5(1):1-41.

4. Scheen AJ. Safety of dipeptidyl peptidase-4 inhibitors for treating type 2 diabetes. Expert Opin Drug Saf. 2015;14(4):505-524.

5. Saisho Y. Alogliptin benzoate for management of type 2 diabetes. Vasc Health Risk Manag. 2015;11:229-243.

6. Berhan A, Berhan Y. Efficacy of alogliptin in type 2 diabetes treatment: a meta-analysis of randomized doubleblind controlled studies. BMC Endocr Disord. 2013;13:9.

7. White WB, Cannon CP, Heller SR, Nissen SE, Bergenstal RM, Bakris GL, Perez AT, et al. Alogliptin after acute coronary syndrome in patients with type 2 diabetes. $\mathrm{N}$ Engl J Med. 2013;369(14):1327-1335.

8. Eliasson B, Moller-Goede D, Eeg-Olofsson K, Wilson C, Cederholm J, Fleck P, Diamant M, et al. Lowering of postprandial lipids in individuals with type 2 diabetes treated with alogliptin and/or pioglitazone: a randomised double-blind placebo-controlled study. Diabetologia. 2012;55(4):915-925.

9. Kim YG, Hahn S, Oh TJ, Park KS, Cho YM. Differences in the HbAlc-lowering efficacy of glucagon-like peptide- 1 analogues between Asians and non-Asians: a systematic review and meta-analysis. Diabetes Obes Metab. 2014;16(10):900-909.

10. Monami M, Lamanna C, Desideri CM, Mannucci E. DPP-4 inhibitors and lipids: systematic review and metaanalysis. Adv Ther. 2012;29(1):14-25.

11. Tanaka T, Higashijima Y, Wada T, Nangaku M. The potential for renoprotection with incretin-based drugs. Kidney Int. 2014;86(4):701-711.

12. Maeda H, Kubota A, Tanaka Y, Terauchi Y, Matsuba I. The safety, efficacy and predictors for HbAlc reduction of sitagliptin in the treatment of Japanese type 2 diabetes. Diabetes Res Clin Pract. 2012;95(1):e20-22.

13. Maeda H, Kubota A, Kanamori A, Tanaka Y, Terauchi Y, Matsuba I. Long-term efficacy and safety of sitagliptin in the treatment of Japanese Type 2 diabetes (ASSET$\mathrm{K} 1)$ to a target of $\mathrm{HbAlc}<7 \%$. J Endocrinol Invest. 2013;36(8):568-573.

14. Kanamori A, Matsuba I. Factors associated with reduced efficacy of sitagliptin therapy: analysis of 93 patients with type 2 diabetes treated for 1.5 years or longer. J Clin Med Res. 2013;5(3):217-221.

15. Kubota A, Maeda H, Kanamori A, Matoba K, Jin Y, Minagawa $\mathrm{F}$, Obana $\mathrm{M}$, et al. Pleiotropic effects of sitagliptin in the treatment of type 2 diabetes mellitus patients. $\mathrm{J}$ Clin Med Res. 2012;4(5):309-313.

16. Maeda H, Kubota A, Kanamori A, Tanaka Y, Terauchi Y, Matsuba I. Effects of sitagliptin on the serum creatinine in Japanese type 2 diabetes. Diabetes Res Clin Pract. 2015;108(3):e42-45. 